\title{
Breast Cancer Presents with a Paraneoplastic Neurologic Syndrome
}

\author{
Pedro Coelho Barata ${ }^{a}$ Joana Morgado ${ }^{b}$ \\ Ana Paula Sousa ${ }^{b}$ Sónia Duarte de Oliveira ${ }^{a}$ \\ Maria Paula Custódio ${ }^{a} \quad$ Lígia Bruno da Costa $^{a}$ \\ José Esteves Pena ${ }^{b}$ \\ ${ }^{a}$ Oncology Department and ${ }^{b}$ Neurology Department, Centro Hospitalar Lisboa \\ Central, Lisboa, Portugal
}

\section{Key Words}

Paraneoplastic neurologic syndromes · Breast cancer - Onconeural antibodies

\begin{abstract}
Background: Paraneoplastic neurologic syndromes (PNS) pose quite an uncommon neurological complication, affecting less than $1 \%$ of patients with breast cancer. Nearly one third of these patients lack detectable onconeural antibodies (ONAs), and improvement in neurologic deficits with concomitant cancer treatments is achieved in less than $30 \%$ of cases. Case Presentation: A 42-year-old, premenopausal woman presented with facial paralysis on the central left side accompanied by a left tongue deviation, an upward vertical nystagmus, moderate spastic paraparesis, dystonic posturing of the left foot, lower limb hyperreflexia and bilateral extensor plantar reflex. After ruling out all other potential neurologic causes, PNS was suspected but no ONAs were found. A PET-CT scan detected increased metabolism in the right breast, as well as an ipsilateral thoracic interpectoral adenopathy. Core biopsy confirmed the presence of an infiltrating duct carcinoma. After breast surgery, the neurologic symptoms disappeared. One week later, the patient was readmitted to the hospital with a bilateral fatigable eyelid ptosis, and two weeks later, there was a noticeable improvement in eyelid ptosis, accompanied by a rapid and progressive development of lower spastic paraparesis. She started adjuvant treatment with chemotherapy with marked clinical and neurological improvement, and by the end of radiotherapy, there were no signs of neurologic impairment.
\end{abstract}

Conclusion: This case study highlights the importance of a high level of vigilance for the detection of PNS, even when ONAs are not detected, as the rapid identification and treatment of the underlying tumor offers the best chance for a full recovery. 


\section{Background}

Paraneoplastic neurologic syndromes (PNS) pose quite an uncommon neurological complication in breast cancer, affecting less than $1 \%$ of patients $[1,2]$. The etiology of PNS remains unknown and is believed to be of an autoimmune origin, resulting from the immune cross-reactivity that occurs between tumor cells and components of the nervous system $[3,4]$. In response to the developing cancer, a patient's immune system produces tumor-directed antibodies known as onconeural antibodies (ONAs). Importantly, there is substantial evidence to suggest that nearly one third of these patients lack detectable ONAs, and improvement in neurologic deficits with concomitant cancer treatments is thus achieved in less than $30 \%$ of patients [5].

\section{Case Presentation}

A 42-year-old, Caucasian, premenopausal woman was admitted to the emergency room, for acute left facial palsy. Fifteen days earlier, she had noticed weakness in her left leg with hypoesthesia but without any changes in sphincter function. A neurological examination revealed facial paralysis on the central left side accompanied by a left tongue deviation, an upward vertical nystagmus, moderate spastic paraparesis, dystonic posturing of the left foot, lower limb hyperreflexia and bilateral extensor plantar reflex. Myoclonus and saccades were both absent. There were no other discernible neurological abnormalities, such as with sensorial perception, sphincter control and cerebellar function. The patient did not experience any disturbances associated with memory, confusion or visual changes. T2-weighted brain magnetic resonance imaging (MRI) revealed small nonspecific white matter lesions (in the periventricular white matter, right corona radiata and bilateral frontal subcortical) without enhancement. The brain computed tomography (CT) was normal. In addition, there were no observable lesions in the spine MRI. Likewise, somatosensory nerve action potentials and cerebrospinal fluid (CSF) analyses were normal. Thus, after ruling out all other potential neurologic causes, PNS was suspected. A full workup, including a complete history and physical exam, chest X-ray, diagnostic bilateral mammogram with ultrasound, chest, abdominal and pelvic CT and a panel of blood tests, which included serum ONAs (anti-Hu, anti-Ri, anti-Yo, anti-Ma and antiamphiphysin) and tumor markers, were performed. No ONAs were found and the investigation did not uncover any primary neoplasm. For this reason, a whole-body positron emission tomographycomputed tomography (FDG-PET/CT) (fig. 1, fig. 2) scan was performed. The FDG-PET/CT detected increased metabolism in both breasts, with greater intensity in the right, as well as an ipsilateral

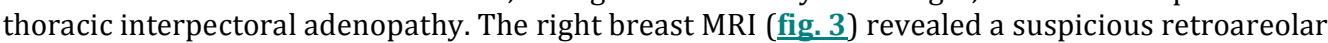
nodule with axillary lymph node involvement. A core biopsy confirmed the presence of an infiltrating duct carcinoma. The patient underwent a right breast lumpectomy with axillary lymph node dissection, which revealed metastases in four lymph nodes $(\mathrm{N})$. The histology results revealed a 1.5$\mathrm{cm}$ high-grade focus, with an invasive ductal carcinoma, pT1c, pN2 $(4 \mathrm{~N}+/ 13 \mathrm{~N})$, positive ER and PR, positive HER-2 (3+, SISH confirmation) and a Ki67 greater than $30 \%$. Following breast-conserving surgery, the neurologic symptoms disappeared. One week later, the patient was readmitted to the hospital with a bilateral fatigable eyelid ptosis. CSF analyses and image scans (brain and spine MRI) did not reveal any abnormalities. In addition, the repetitive nerve stimulation and single-fiber EMG results were normal and the anti-acetylcholine receptor antibodies were negative. Intravenous immunoglobulin therapy was performed, but it did not yield any results. Two weeks after admission, there was a noticeable but slight improvement in eyelid ptosis, which was accompanied by a rapid and progressive development of lower spastic paraparesis. Four weeks after surgery, the patient started adjuvant chemotherapy, consisting of four cycles of the AC protocol (cyclophosphamide and doxorubicin), followed by 4 cycles of docetaxel, once every 3 weeks, in association with trastuzumab, every 3 weeks for a total of 18 cycles. At the end of chemotherapy, the patient showed marked clinical and neurological improvement. After chemotherapy, the patient began tamoxifen, and sequential radiotherapy was performed. By the end of the radiotherapy, there were no signs of neurologic impairment. The patient was reviewed by our Oncology Department, once the trastuzumab protocol was completed. The T2-weighted brain MRI was repeated and revealed the same nonspecific white 
matter lesions observed previously. The patient was in complete clinical remission without symptoms and any neurological disturbances and was leading a normal life.

\section{Discussion}

According to the Paraneoplastic Neurological Syndrome Euronetwork diagnostic criteria [6], our patient fulfilled the requirements for the clinical diagnosis of possible non-classical PNS. The first time our patient was admitted to the hospital, she presented with neurologic symptoms that were characterized by facial paralysis of the central left side, left tongue deviation with an upward vertical nystagmus, moderate spastic paraparesis, dystonic posturing of the left foot, lower limb hyperreflexia and bilateral extensor plantar reflex. All of these symptoms may include more than one level of motor involvement, with both the spinal cord and brainstem being engaged. Facial paralysis, tongue deviation and vertical nystagmus are consistent with brainstem encephalitis, which is present in one third of patients with paraneoplastic encephalomyelitis (PEM); the clinical presentation of PEM reflects the distribution of this multifocal inflammatory condition that is associated with remote neoplasia. Neuroimaging studies of PEM using MRI may show increased signal intensity on the T2weighted scan, which is consistent with the inflammatory process. However, certain neuroimaging studies are standard, as reported in the present case study [7]. Given that the CSF examination was normal, the proposed diagnosis was less likely, as CSF may show elevated white cell counts, oligoclonal bands, and intrathecal synthesis of IgG, indicating an immune-mediated or inflammatory etiology [3].

A second neurological examination following surgery revealed a bilateral fatigable eyelid ptosis, suggesting a neuromuscular postsynaptic disorder such as myasthenia gravis (MG). The link of MG with tumors other than thymoma remains controversial, although some studies have shown that breast cancer is the most common among extrathymic malignancies [8]. Evidence suggests that the mechanism of neuronal attack in cancer is immune-mediated, which might explain how a second neurological relapse occurred following surgery of breast. According to the literature, the accuracy of the immunoprecipitation assay for the detection of almost all onconeural antibodies may be superior in the former assay [9]. In our particular case study, ONAs were tested with immunofluorescence using patient serum samples and cannot be detected. Further studies are most likely needed to develop more sensitive assays, as the failure to detect onconeural antibodies in a patient with suspected PNS has important consequences for the treatment of the patient.

\section{Conclusion}

This case study highlights the importance of maintaining a high level of vigilance for the detection of PNS, even when ONAs are not detected, as the rapid identification and treatment of the underlying tumor offers the best chance for both a full recovery and the prevention of any further neurologic impairment. 


\section{Consent}

Written informed consent was obtained from the patient for publication of this case report and accompanying images. A copy of the written consent is available for review by the Editor-in-Chief of this journal.

\section{Acknowledgements}

We thank Flávia Pinto who helped with acquisition of data and administrative work.

\section{Disclosure Statement}

The authors declare that they have no competing interests.

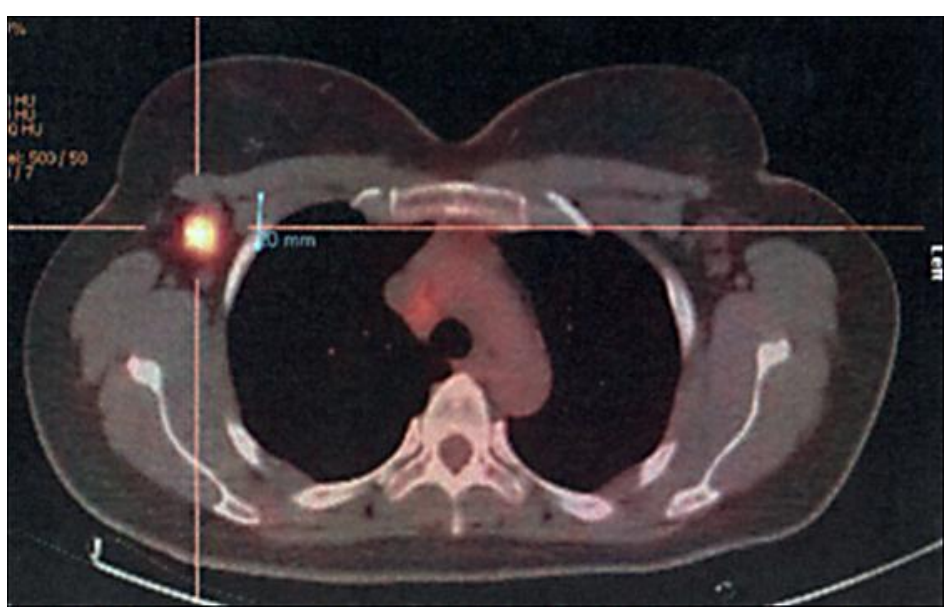

Fig. 1. FDG-PET/CT detected increased metabolism in the right breast. 


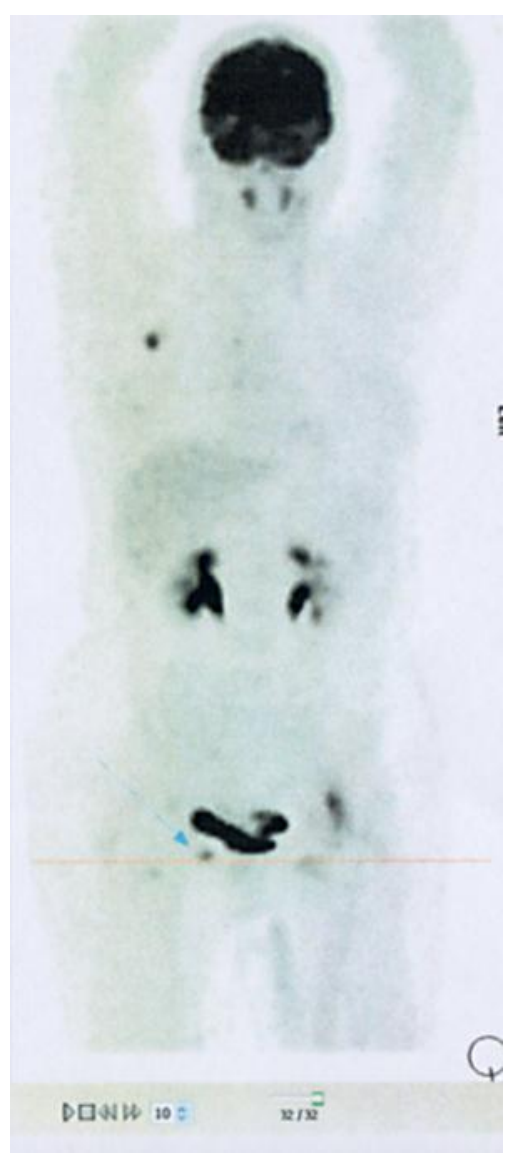

Fig. 2. Right thoracic interpectoral adenopathy detected by FDG-PET/CT. 


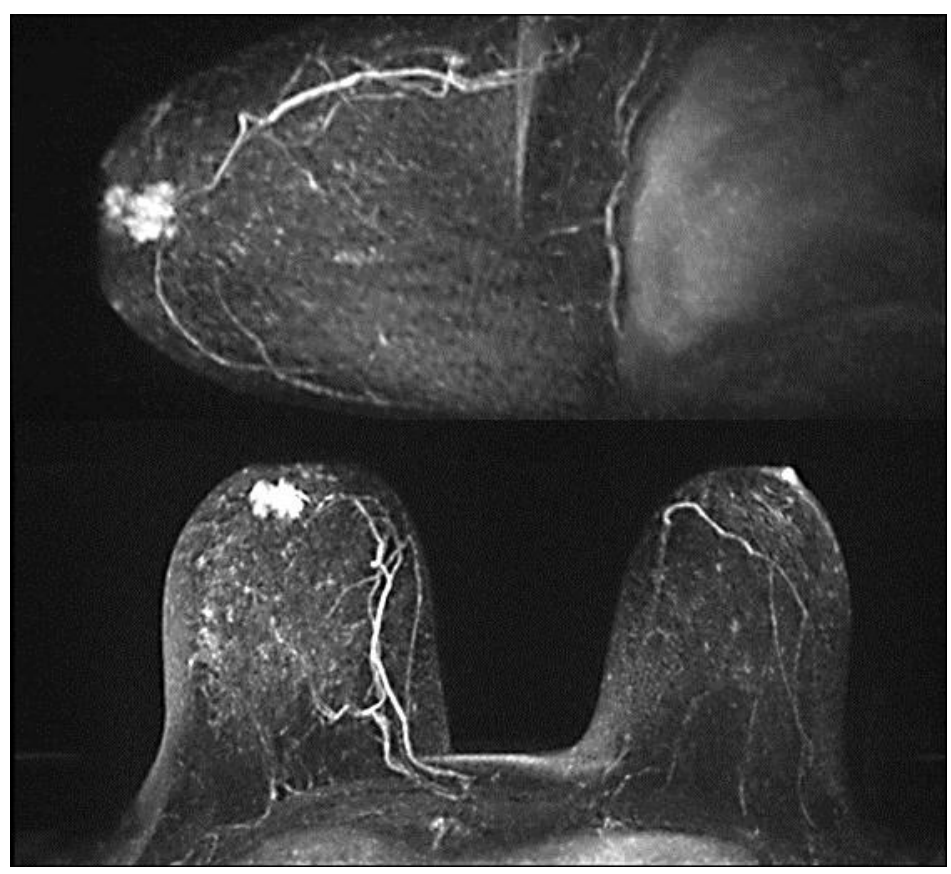

Fig. 3. Suspicious retroareolar nodule of the right breast detected by breast MRI.

\section{References}

1 Gatti G, Simsek S, Kurne A: Paraneoplastic neurological disorders in breast cancer. Breast 2003;12:203207.

$\checkmark 2$ Kawasoe T, et al: A case report of paraneoplastic neurologic syndrome associated with occult breast cancer. Breast Cancer 2006;13:2.

3 de Beukelaar J, Sillevis Smitt PA: Managing paraneoplastic neurological disorders. Oncologist 2006;11:292-305.

-4 Storstein A, Vedeler C: Paraneoplastic neurological syndromes and onconeural antibodies: clinical and immunological aspects. Adv Clin Chem 2007;44:143-185.

5 Pelosof LC, Gerber DE: Paraneoplastic syndromes: an approach to diagnosis and treatment. Mayo Clin Proc 2010;85:838-854.

6 Graus F, Delattre J, Antoine J, et al: Recommended diagnostic criteria for paraneoplastic neurological syndromes. J Neurol Neurosurg Psychiatry 2004;75:1135-1140.

7 Lim KE, Hsu YY, Hsu WC, Chan CY: Multiple complete ring-shaped enhanced MRI lesions in acute disseminated encephalomyelitis. Clin Imaging 2003;27:281-284.

$>8$ Dalmau J, Gultekin HS, Posner JB: Paraneoplastic neurologic syndromes: pathogenesis and physiopathology. Brain Pathol 1999;9:275.

-9 Storstein A, Monstad SE, Haugen M, et al: Onconeural antibodies: improved detection and clinical correlations. J Neuroimmunol 2011;232:166-170. 